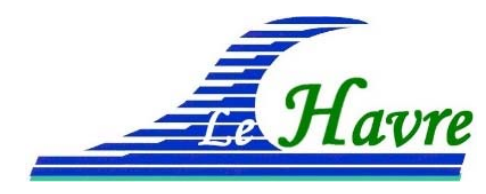

XVI ${ }^{\text {èmes }}$ Journées Nationales Génie Côtier - Génie Civil
Le Havre, 2020

DOI:10.5150/jngcgc.2020.015 (C) Editions Paralia CFL

disponible en ligne - http://www.paralia.fr - available online

\title{
Hydrodynamique de la zone pré-littorale en conditions d'états de mer énergétiques
}

\author{
Marc PEZERAT ${ }^{1}$, Xavier BERTIN ${ }^{1}$, Baptiste MENGUAL ${ }^{1}$, \\ Thibault COULOMBIER ${ }^{1}$, Denis DAUSSE ${ }^{1}$
}

\author{
1. UMR 7266 LIENSs, CNRS - Université de La Rochelle, \\ 2 rue Olympe de Gouges, 17000 La Rochelle, France. \\ marc.pezerat@univ-lr.fr
}

\section{Résumé :}

Cette étude présente les résultats d'une campagne de mesures réalisée en octobre 2019 à l'entrée du pertuis d'Antioche, entre les îles de Ré au Nord et d'Oléron au Sud (France), au moment du passage de la tempête Lorenzo au large du Golfe de Gascogne. Les données expérimentales sont complétées par l'application d'un système de modélisation couplé vague-courant en $3 \mathrm{D}$. Les résultats tendent à montrer que l'effet du couplage a relativement peu d'impact sur les courants moyens, en revanche la distribution verticale des vitesses horizontales est mieux reproduite lorsque le couplage est activé : une réduction de l'erreur quadratique moyenne de l'ordre de 15 à $40 \%$ selon le cycle de marée est observée. De plus, l'effet des vagues sur les courants le long de la colonne d'eau semble d'autant plus important que les vagues incidentes sont énergétiques.

\section{Mots-clés :}

Interactions vagues-courant, Zone pré-littorale, Vague de tempête, Rugosité.

\section{Introduction}

La zone pré-littorale correspond à la partie interne du plateau continental qui s'étend du point de déferlement des vagues jusqu'à des profondeurs de l'ordre de 20 à $30 \mathrm{~m}$. Cette partie de la bande côtière est exploitée de façon importante par l'Homme, comme par exemple pour la pêche, l'extraction de granulats ou encore l'installation de systèmes d'énergies marines renouvelables. En outre, l'hydrodynamique de la zone pré-littorale contrôle les échanges sédimentaires entre la côte et le large. Les courants de marée étant généralement faibles le long des côtes ouvertes, les vagues ont un rôle essentiel sur la dynamique hydro-sédimentaire (IDIER et al., 2006), a fortiori lors des tempêtes. Compte tenu des difficultés techniques liées à l'instrumentation dans de telles conditions, la plupart des études reposent sur des simulations numériques, dont les résultats ne sont pas validés en présence de vagues de tempête. Nous proposons ici d'étudier l'effet des vagues sur les courants dans la colonne d'eau en comparant deux formulations de la contrainte de friction au fond. GANJU \& SHERWOOD (2010) ont notamment montré que la prise en compte des interactions vagues-courant dans le calcul de la contrainte au fond n'améliorait pas significativement les performances de leur modèle comparé à une 


\section{Thème 1 - Hydrodynamique côtière}

rugosité uniforme. Cependant leur étude a été réalisée en présence de vagues modérément énergétiques. Cet article présente une campagne de mesures organisée en octobre 2019 au nord-ouest de l'île d'Oléron (France) en présence de vagues de tempête associées au passage de l'ouragan Lorenzo au large du Golfe de Gascogne. L'étude des mesures acquises est complétée par des simulations numériques avec le système de modélisation SCHISM (ZHANG et al., 2016), qui réalise le couplage entre un modèle de circulation hydrodynamique 3D et le modèle spectral de propagation des vagues WWM (ROLAND et al., 2012).

\section{Matériel et méthodes}

\subsection{Zone d'étude}

La zone étudiée se situe à l'entrée du pertuis d'Antioche entre les îles de Ré au Nord et d'Oléron au Sud (figure 1a). Le régime de marée est semi-diurne et le marnage varie entre $1.1 \mathrm{~m}$ en mortes-eaux et $5.5 \mathrm{~m}$ en vives-eaux (DODET et al., 2019). Le climat de vague est caractérisé par une hauteur significative $\left(H_{m 0}\right)$ de $1.6 \mathrm{~m}$ en moyenne au niveau de l'isobathe $30 \mathrm{~m}$ (DODET et al., 2019) et peut atteindre $10 \mathrm{~m}$ au large pendant les tempêtes hivernales (NICOLAE LERMA et al., 2015). Les houles proviennent principalement de directions Ouest à Sud-Ouest, ainsi l'entrée du pertuis d'Antioche est directement exposée aux houles incidentes. Le dispositif de mesure a été déployé entre le 3 et le 6 octobre 2019 à l'Ouest de la zone d'extraction de granulats marin de Chassiron (figure 1b), partagée en 4 fosses d'extraction (dont 3 sont principalement exploitées), afin que les vagues incidentes mesurées ne soient pas transformées par l'anomalie bathymétrique.
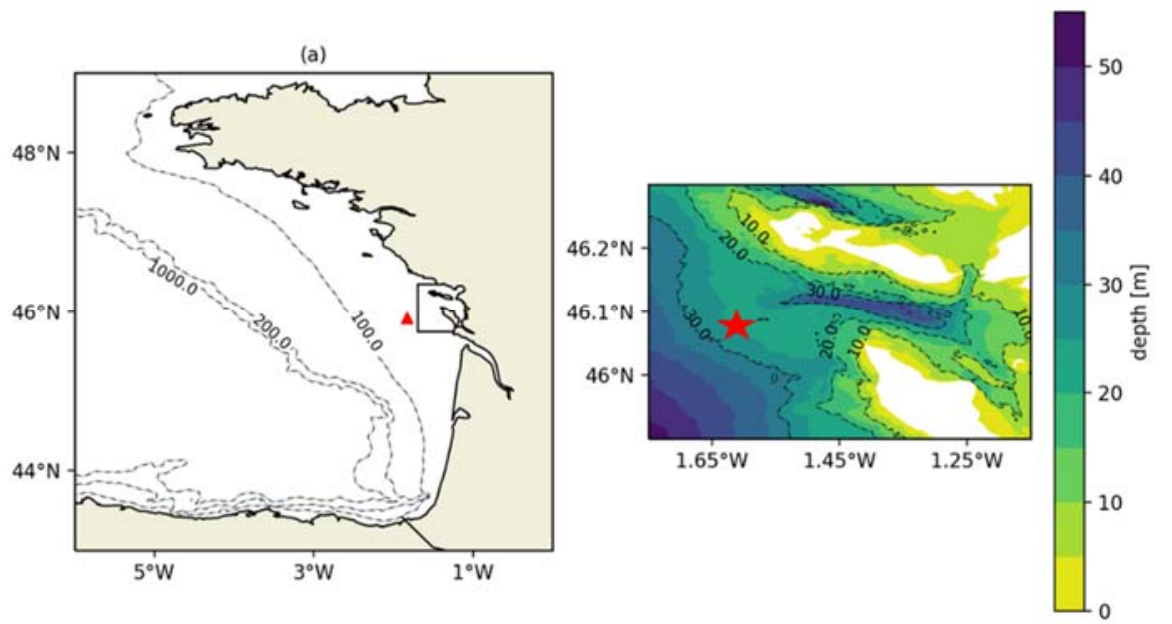

Figure 1. (a) Localisation de la zone d'étude et position de la bouée "Oléron Large" (triangle rouge). (b) Zoom sur le pertuis d'Antioche et position du dispositif de mesure (étoile rouge). La bathymétrie est référencée par rapport au niveau moyen. 


\section{XVI'mes Journées Nationales Génie Côtier - Génie Civil \\ Le Havre 2020}

\subsection{Dispositif de mesures et traitement des données}

Afin de mesurer les courants sur toute la hauteur de colonne d'eau, un dispositif innovant a été mis en place. Deux profileurs acoustiques ont été montés sur une même cage d'environ $0.7 \mathrm{~m}$ de hauteur déployée au fond. L'un est orienté vers la surface (ADCP $600 \mathrm{kHz}$ ) et le second vers le fond (ADCP $2 \mathrm{MHz}$ ). Pour les deux instruments, les acquisitions de mesures s'alternent entre un cycle "courant", où des profils verticaux de vitesse et la pression au fond sont moyennés sur 10 minutes, et un cycle "vague", où les vitesses dans la cellule du fond et la pression sont mesurées à $2 \mathrm{~Hz}$ pendant 20 minutes permettant ainsi de calculer les paramètres intégraux des vagues (nous y reviendrons ciaprès). Pour les mesures de courants, la résolution verticale des cellules est de $0.5 \mathrm{~m}$ pour l'ADCP à $600 \mathrm{kHz}$ et de $0.1 \mathrm{~m}$ pour l'ADCP à $2 \mathrm{MHz}$.

Les échantillons de mesures de pression acquis par l'ADCP $600 \mathrm{kHz}$ ont d'abord été corrigés de la pression atmosphérique, puis le signal d'élévation de la surface libre a été reconstruit en utilisant la méthode de reconstruction linéaire de BISHOP \& DONELAN (1987). Cette méthode requiert l'utilisation d'une fréquence de coupure haute pour s'affranchir du bruit du capteur, fixée dans notre cas à $0.2 \mathrm{~Hz}$. Pour chaque échantillon, le spectre d'élévation $E(f)$ a ensuite été calculé en moyennant les spectres obtenus par transformée de Fourier sur 10 fenêtres de Hann se chevauchant de moitié. Enfin, les paramètres intégraux sont calculés grâce aux moments de chaque spectre :

$$
H_{m 0}=4 \sqrt{m_{0}} ; T_{m 02}=\sqrt{\frac{m_{0}}{m_{2}}} \text { avec } m_{p}=\int_{f_{\text {min }}}^{f_{\text {max }}} f^{p} E(f) d f
$$

En cohérence avec la fréquence de coupure utilisée pour la reconstruction de la surface libre, $f_{\max }$ a été fixé à $0.2 \mathrm{~Hz}$ et $f_{\min }=0.04 \mathrm{~Hz}$. Nous disposons également des spectres d'élévation mesurés par la bouée "Oléron Large" déployée au large de l'île d'Oléron dans le cadre du réseau CANDHIS. Les paramètres intégraux ont été calculés de la même manière en étendant l'intervalle d'intégration jusqu'à $0.4 \mathrm{~Hz}$.

\subsection{Présentation du système de modélisation}

Les modèles de vagues et de circulation 3D sont couplés en utilisant le formalisme de force vortex tel que décrit par GUERIN et al. (2018). Alternativement, les deux modèles peuvent être découplés. Les deux modèles s'appuient sur le même maillage non structuré et utilisent la même décomposition du domaine. La discrétisation verticale du modèle de circulation s'appuie sur une grille S-Z composée de 21 niveaux. Le modèle de vague est forcé à la frontière ouverte avec des spectres issus d'une application sur l'Atlantique Nord du modèle spectral WaveWatch III (WWIII ; TOLMAN, 1991) en utilisant un maillage structuré résolu à $0.2^{\circ}$. Le forçage tidal imposé au modèle de circulation intègre 19 harmoniques de marée interpolées depuis le modèle régional de BERTIN et al. (2012). Le forçage atmosphérique utilisé pour le modèle couplé SCHSIM/WWM comme pour WWIII se compose de la pression atmosphérique au niveau moyen et du vent à $10 \mathrm{~m}$ issus 


\section{Thème 1 - Hydrodynamique côtière}

du rejeu du Climate Forecast System Reanalysis - CFSR (SAHA et al., 2014). Pour les deux modèles de vagues, le terme source lié au vent et la dissipation par moutonnement sont formulés d'après ARDHUIN et al. (2010) et les interactions non linéaires entre quadruplets sont prises en compte selon l'approche de HASSELMAN \& HASSELMAN (1985). En eau peu profonde, trois autres processus de transformations des vagues sont considérés: la friction sur le fond (HASSELMAN et al., 1973), le déferlement bathymétrique (BATTJES \& JANSSEN, 1978) et les interactions non linéaires entre triades (ELDEBERKY, 1996). La rugosité physique (zo) varie spatialement selon la nature du fond entre $0.5 \mathrm{~mm}$ au-delà du seuil interinsulaire et $5 \mathrm{~mm}$ au niveau des zones rocheuses autour de l'extrémité Nord de l'île d'Oléron d'après BERTIN et al. (2020). Enfin, comme la turbulence induite par les vagues proche du fond modifie la rugosité ressentie par le courant, une rugosité apparente zoa est introduite dans le modèle couplé vague-courant selon l'approche de MADSEN (1994). Celle-ci prévaut en dehors de la couche limite turbulente induite par les vagues à l'intérieure de laquelle la rugosité physique est considérée.

\section{Résultats}

Dans cette partie les résultats obtenus en utilisant une configuration couplée ou découplée du modèle sont comparés aux mesures. Les performances du modèle sont quantifiées par l'erreur quadratique moyenne normalisée (NRMSE) :

$$
\operatorname{NRMSE}(X)=\frac{\sum_{i=1}^{N}\left(\hat{X}_{i}-X_{i}\right)^{2}}{\sum_{i=1}^{N} X_{i}{ }^{2}} \times 100
$$

Avec $X$ et $\hat{X}$ les vecteurs des grandeurs mesurées et des grandeurs modélisées correspondantes de tailles $N$, respectivement.

\subsection{Validation du modèle : vagues, courants intégrés et élévation de la surface libre}

Les performances du modèle de vague sont estimées en comparant les paramètres intégraux calculés avec les spectres issus du modèle, intégrés sur le même intervalle que les observations. Les résultats à l'ADCP du modèle couplé sont proches de ceux du modèle découplé ce qui tend à montrer que localement les courants affectent peu la propagation des vagues (figure 2). Les erreurs sur $H_{m 0}, T_{m 02}$ et $T_{p}$ atteignent respectivement $20 \%, 8 \%$ et $15 \%$. L'erreur relativement élevée sur $H_{m 0}$ est imputable à un biais positif au pic de la tempête (autour du 04/10 - 12h) qui atteint environ $1 \mathrm{~m}$. Les résultats du modèle semblent montrer que le shoaling l'emporte sur les processus dissipatifs ce que l'on ne retrouve pas dans les observations (comparer figures $2 \mathrm{a}$ et $2 \mathrm{~b}$ ). Une perspective d'amélioration des résultats qui consiste à affiner la paramétrisation de la friction sur le fond est en cours d'investigation. En outre, le choix de la fréquence de coupure $f_{\max }$ relativement conservative impose de s'affranchir des composantes hautes fréquences du spectre. La période moyenne $T_{m 02}$ s'en trouve améliorée lorsqu'on la compare au résultat obtenu à la bouée "Oléron Large" où l'on constate une surestimation 


\section{XVIèmes Journées Nationales Génie Côtier - Génie Civil \\ Le Havre 2020}

de l'ordre $2 \mathrm{~s}$ imputable à une mauvaise représentation de la mer de vent (comparer figures $2 \mathrm{c}$ et $2 \mathrm{~d}$ ).

Les variations de hauteur d'eau modélisées sont identiques pour les deux configurations $\mathrm{du}$ modèle et en bonne adéquation avec les observations (NRMSE $=12 \%$ ). Pour le courant moyenné sur la colonne d'eau, les deux configurations conduisent à des résultats similaires. Comparés aux observations, on constate une surestimation du courant modélisé aux étales de l'ordre de $6 \mathrm{~cm} / \mathrm{s}$ et dans une moindre mesure au jusant. Ces erreurs sont relativement proches de la précision du capteur de l'ordre de $2 \mathrm{~cm} / \mathrm{s}$. L'erreur quadratique moyenne atteint $24 \%$ avec le modèle couplé et $21 \%$ avec le modèle découplé, figure 3.

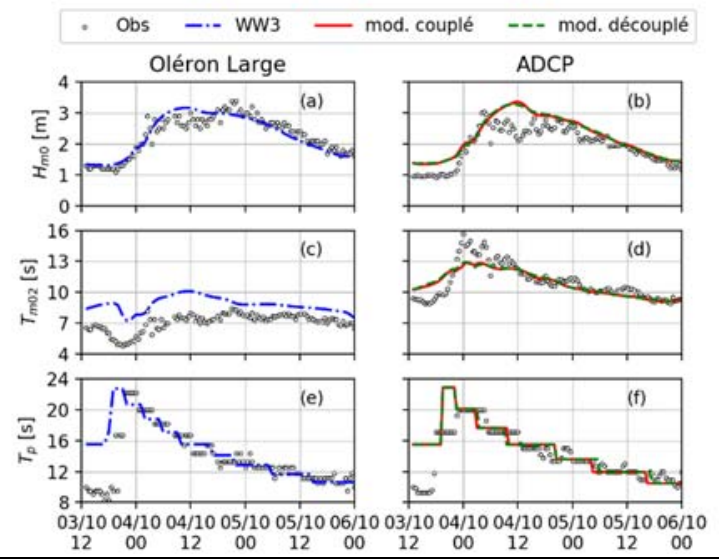

Figure 2. Comparaison modèles/observations des paramètres intégraux des vagues à la bouée "Oléron Large" (panneaux à gauche) et à l'ADCP (panneaux à droite).

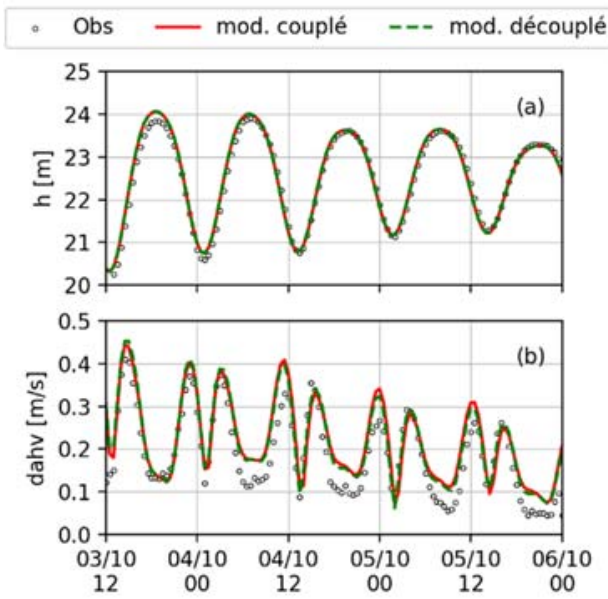

Figure 3. Comparaison modèles/observations de la hauteur d'eau (a) et de la norme du courant horizontal moyenné sur la colonne d'eau (b). Un décalage de $1 \mathrm{~m}$ imputable à la donnée bathymétrique a été corrigé sur la hauteur d'eau modélisée. 


\section{Thème 1 - Hydrodynamique côtière}

3.2 Effet des vagues sur la distribution des vitesses horizontales dans la colonne d'eau Les courants dans la colonne d'eau sont qualitativement bien représentés par les deux configurations du modèle comme le montre la figure 4. Afin d'estimer quantitativement l'effet du couplage, quatre profils ont été extraits pour deux épisodes de flot et deux épisodes de jusant sous des climats de vagues contrastés (symbolisés par les traits verticaux sur la figure 4). Les résultats obtenus avec le modèle couplé sont meilleurs et l'erreur est d'autant plus réduite que le climat de vague est énergétique comme le montre la comparaison des profils bleus et mauves, figure 5. En outre, quelle que soit la configuration employée, l'erreur quadratique moyenne est plus élevée pour les profils extraits au jusant.

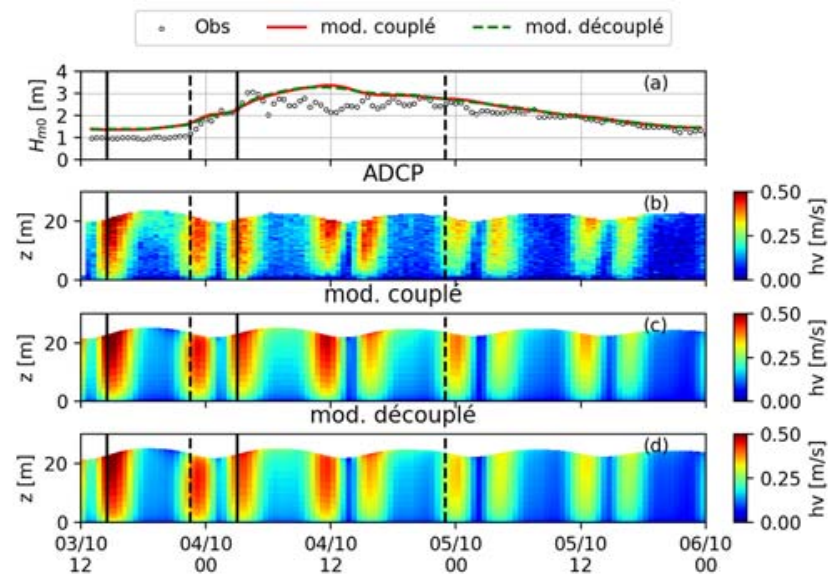

Figure 4. Courants mesurés (b) et modélisés (c et d). La série temporelle de $H_{m 0}$ est représentée à titre indicatif (a). Un masque a été appliqué aux mesures au-delà d'une distance équivalente à la hauteur d'eau à laquelle la demi-hauteur significative des vagues a été retranchée. Les traits verticaux symbolisent les profils extraits.

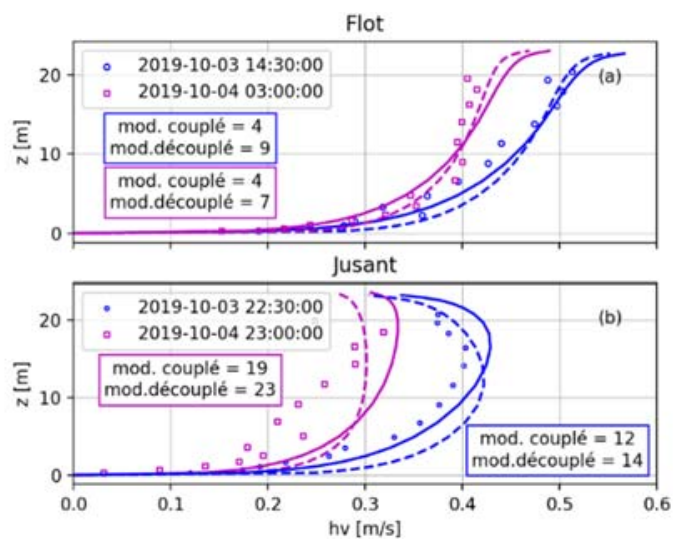

Figure 5. Comparaison modèle/observation des profils de vitesse extraits au flot (a) et au jusant (b). Les traits pleins (resp. pointillés) correspondent aux résultats obtenus avec le modèle couplé (resp. découplé). Les erreurs quadratiques moyennes correspondantes sont données dans les encadrés. 


\section{XVI'̀mes Journées Nationales Génie Côtier - Génie Civil \\ Le Havre 2020}

\section{Conclusions}

La campagne de terrain réalisée lors du passage de l'ouragan Lorenzo au large du Golfe de Gascogne a permis de tester un dispositif innovant afin de pouvoir mesurer les courants sur l'intégralité de la colonne d'eau. L'analyse des observations et des résultats numériques montre l'effet non négligeable des vagues sur les courants dans la colonne d'eau, a fortiori lorsque les vagues incidentes sont énergétiques. Bien que ces effets aient finalement peu d'influence sur les courants moyens, une paramétrisation adéquate de la rugosité dans un modèle couplé vague-courant apparait souhaitable en particulier si l'on envisage une application dans un modèle morphodynamique. Une nouvelle campagne de mesures devrait être mise en œuvre dans des conditions similaires avec des équipements supplémentaires afin d'étudier les processus de transformation des vagues et l'impact sur la circulation hydrodynamique. Un couplage avec le modèle de transport sédimentaire et d'évolution du fond SED3D (PINTO et al., 2012) sera mis en place afin d'étudier les évolutions morphodynamiques.

\section{Remerciements}

L'auteur principal est cofinancé par la CDA de La Rochelle et le projet FEDER DURALIT. Ces travaux de recherche ont été menés dans un programme de Chaire Régionale EVEX.

\section{Références bibliographiques}

ARDHUIN F., ROGERS E., BABANIN A.V., FILIPOT J.-F., MAGNE R., ROLAND A., VAN DER WESTHUYSEN A., QUEFFEULOU P., LEFEVRE J.-M., AOUF L., COLLARD F. (2010). Semiempirical dissipation source functions for ocean waves. Part I: Definition, calibration, and validation. Journal of Physical Oceanography, vol. 40 (9), pp 1917-1941. https://doi.org/10.1175/2010JPO4324.1

BATTJES J.A., JANSSEN J.P.F.M. (1978). Energy loss and set-up due to breaking of random waves. $16^{\text {th }}$ International Conference on Coastal Engineering. pp 569-587. https://doi.org/10.1061/9780872621909.034

BERTIN X., BRUNEAU N., BREILH J-F., FORTUNATO A.B., KARPYTCHEV M. (2012). Importance of wave age and resonance in storm surges: The case Xynthia, Bay of Biscay. Ocean Modelling, vol. 42, pp 16-30. https://doi.org/10.1016/j.ocemod.2011.11.001 BERTIN X., MENGUAL B., MARTINS K., et al. (2020). Recent advances in tidal inlet morphodynamics. In: Malvárez, G. and Navas, F. (eds.), Proceedings from the International Coastal Symposium, ICS 2020, Seville, Spain. Journal of Coastal Research, Special Issue No. 95, pp 1-5. In press

BISHOP C.T., DONELAN M.A. (1987). Measuring waves with pressure transducers. Coastal Engineering, Vol. 11(4), pp 309-328. https://doi.org/10.1016/0378-3839(87)90031-7 


\section{Thème 1 - Hydrodynamique côtière}

DODET G., BERTIN X., BOUCHETTE F., GRAVELlE M., TESTUT L., WÖPPELMANN G. (2019). Characterization of sea-level variations along the metropolitan coasts of France: waves, tides, storm surges and long-term changes. Journal of Coastal Research, 2019, Vol. 88, nº sp1, pp 10-24. https://doi.org/10.2112/SI88-003.1

ELDEBERKY Y. (1996). Nonlinear transformation of wave spectra in the nearshore zone. Ph.D., Delft University of Technology, 223p. http://resolver.tudelft.nl/uuid:707ca57d-81c34103-bc6e-aae1c90fce63

GANJU N.K., SHERWOOD C.R. (2010). Effect of roughness formulation on the performance of a coupled wave, hydrodynamic, and sediment transport model. Ocean Modelling, Vol. 33(3-4), pp 299-313. https://doi.org/10.1016/j.ocemod.2010.03.003

GUERIN T., BERTIN X., COULOMBIER T., DE BAKKER A. (2018). Impacts of waveinduced circulation in the surf zone on wave setup. Ocean Modelling, 2018, vol. 123, pp 86-97. https://doi.org/10.1016/j.ocemod.2018.01.006

HASSELMANN, K., BARNETT, T. P., BOUWS, E., CARLSON H., CARTWRIGHT D.E., ENKE K., EWING J.A., GIENAPP H., HASSELMANN D.E., KRUSEMAN P., MEERBURG A., MÜLLER P., OLBERS D.J., RICHTER K., SELL W., WALDEN H. (1973). Measurements of wind-wave growth and swell decay during the Joint North Sea Wave Project (JONSWAP). Ergänzungsheft, pp 8-12.

HASSELMANN S., HASSELMANN, K. (1985). Computations and parameterizations of the nonlinear energy transfer in a gravity-wave spectrum. Part I: A new method for efficient computations of the exact nonlinear transfer integral. Journal of Physical Oceanography, Vol. 15(11), pp 1369-1377. https://doi.org/10.1175/15200485(1985)015<1369:CAPOTN >2.0.CO;2

IDIER D., PEDREROS R., OLIVEROS C., SOTTOLICHIO A., CHOPPIN L., BERTIN $\mathrm{X}$. (2006) Contributions respectives des courants et de la houle dans la mobilité sédimentaire d'une plate-forme interne estuarienne. Exemple: le seuil interinsulaire, au large du pertuis d'Antioche, France. Comptes Rendus Geoscience, Vol. 338(10), pp 718726. https://doi.org/10.1016/j.crte.2006.05.004

NICOLAE LERMA A., BULTEAU T., LECACHEUX S., IDIER D. (2015). Spatial variability of extreme wave height along the Atlantic and channel French coast. Ocean Engineering, Vol. 97, pp 175-185. https://doi.org/10.1016/j.oceaneng.2015.01.015

MADSEN O.S. (1994). Spectral wave-current bottom boundary layer flows. $24^{\text {th }}$ Intl. Conference on Coastal Engineering. pp 384-398. https://doi.org/10.1061/9780784400890.030 PINTO L., FORTUNATO A.B., ZHANG Y., OLIVEIRA A., SANCHO F.E.P. (2012). Development and validation of a three-dimensional morphodynamic modelling system for non-cohesive sediments. Ocean Modelling, Vol. 57, pp 1-14. https://doi.org/10.1016/j.ocemod.2012.08.005

ROLAND A., ZHANG Y.J., WANG H.V., MENG Y. Q., TENG Y.-C., MADERICH V., BROVCHENKO I., DUTOUR-SIKIRIC M., ZANKE U. (2012). A fully coupled 3D 


\section{XVIèmes Journées Nationales Génie Côtier - Génie Civil \\ Le Havre 2020}

wave-current interaction model on unstructured grids. Journal of Geophysical Research: Oceans, Vol. 117, $\mathrm{n}^{\circ} \mathrm{C} 11$. https://doi.org/10.1029/2012JC007952

SAHA S., MOORTHI S., WU X., WANG J., NADIGA S., TRIPP P., BEHRINGER D., HOU Y.-T., CHUANG H.Y,IREDELL M., EK M., MENG J., YANG R. Q., PEÑA MENDEZ M., VAN DEN DOOL H., ZHANG Q., WANG W. Q., CHEN M. Y., BECKER E. (2014). The NCEP climate forecast system version 2. Journal of climate, Vol. 27(6), pp 2185-2208. https://doi.org/10.1175/JCLI-D-12-00823.1

TOLMAN H.L. (1991). A third-generation model for wind waves on slowly varying, unsteady, and inhomogeneous depths and currents. Journal of Physical Oceanography, Vol. 21(6), pp 782-797. https://doi.org/10.1175/1520-0485(1991)021<0782:ATGMFW>2.0.CO;2 ZHANG Y.J., YE F., STANEV E.V., GRASHORN S. (2016). Seamless cross-scale modeling with SCHISM. Ocean Modelling, Vol. 102, pp 64-81. https://doi.org/10.1016/j.ocemod.2016.05.002 
Thème 1 - Hydrodynamique côtière 